\title{
Estudo Retrospectivo do Resultado Anatomopatológico de 100 Polipectomias Colonoscópicas Realizadas na FMB-UNESP
}

\author{
Retrospective study of the anatomopathological results of 100 colonoscopic \\ polypectomies performed at FMB-UNESP
}

\author{
ROGÉRIO SAAD HOSSNE ; MARAFERNANDES MARANHÃO²; FLÁVIOAUGUSTO DE CARVALHO; FABIANA \\ GUANDALINI MENDES ${ }^{2}$
}

${ }^{1}$ Professor Assistente Doutor da Faculdade de Medicina de Botucatu - UNESP; ${ }^{2}$ Aluno(a) do $6^{\circ}$ ano Médico da Faculdade de Medicina de Botucatu - UNESP.

HOSSNE RS; MARANHÃO MF; CARVALHO FA; MENDES FG. Estudo Retrospectivo do Resultado Anatomopatológico de 100 Polipectomias Colonoscópicas Realizadas na FMB-UNESP. Rev bras Coloproct, 2007;27(3): 251-255.

RESUMO: Objetivo: Analisar retrospectivamente o resultado do estudo anatomopatológico de polipectomias colonoscópicas realizadas no Setor de Endoscopia da Faculdade de Medicina de Botucatu - UNESP durante os anos de 2002 e 2003. Material e Métodos: Estudamos retrospectivamente, a partir de procedimentos colonoscópicos realizados em nosso serviço, as características dos pólipos retirados: tamanho, localização e distribuição, bem como o motivo da indicação do mesmo e o resultado do estudo anatomopatológico. Resultados: Num total de 100 polipectomias colonoscópicas realizadas em 75 pacientes, observamos que $63 \%$ dos pólipos localizava-se em reto e sigmóide e 15,8\% no cólon descendente; o estudo anátomopatologico evidenciou que a grande maioria tinha características adenomatosas (54\%). A idade dos pacientes variou entre 6 e 92 anos, não havendo predominância quanto ao sexo; a principal indicação para a realização de colonoscopia foi sangramento. Conclusão: Constatamos o importante papel diagnóstico e terapêutico das colonoscopias no que diz respeito ao câncer colorretal; os achados demonstram que, em nossa casuística, a maioria os pólipos colônicos são adenomatosos, pequenos e de localização distal.

Descritores: pólipos do colo, pólipos adenomatosos, colonoscopia, neoplasias colorretais, histologia.

\section{INTRODUÇÃO}

O estudo anatomopatológico e genético dos pólipos colônicos, nos últimos tempos, tem se tornado cada vez mais importante; sabe-se que é elevada a prevalência de pólipos colônicos, principalmente os assintomáticos, e sua correlação com a incidência de câncer colorretal. A sequiência adenoma-carcinoma é a principal causa do câncer colorretal e correlacionase com o aumento do número de casos; estudos das bases moleculares e genéticas desta sequiência amplamente estudados, serve de modelo no estudo da

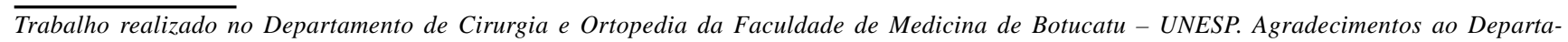
mento de Patologia. 
carcinogênese, merecendo hoje, cuidados à parte ${ }^{(1)}$. Além disso, sabemos que, entre os pólipos que se iniciam com características adenomatosas, o componente arquitetural viloso presente na sua estrutura acarreta maior risco de progressão para malignidade ${ }^{(2)}$.

No entanto, a classificação dos pólipos do cólon e reto não envolve somente o tipo adenomatoso, como também os inflamatórios, hiperplásicos e juvenis $^{(3)}$. Postula-se que entre estes, os hiperplásicos também possam levar ao início de um processo neoplásico focal $^{(4,5)}$.

No que diz respeito ao real papel terapêutico da retirada dos pólipos hiperplásicos e adenomatosos, os dados obtidos na literatura ${ }^{(1)}$ ressaltam a importância deste procedimento, pois o mesmo impede a progressão da sequiência adenoma-carcinoma, favorecendo a prevenção. Estes dados apontam para o fato de que aproximadamente $30 \%$ dos carcinomas observados por colonoscopia ou retirados cirurgicamente coexistiam com adenomas no mesmo segmento. Sabese ainda que ambas as lesões são encontradas mais freqüentemente no cólon distal à flexura esplênica fortalecendo teoria da progressão ${ }^{(6)}$. É importante salientar também que estes pólipos têm prevalência aumentada conforme avança a idade do paciente ${ }^{(6,7)}$. Não é o propósito deste estudo discutir possíveis implicações ambientais de hábitos e dieta na gênese dos cânceres de intestino grosso, apesar de sabermos que a dieta, conjuntamente com os fatores ambientais, influenciam no aparecimento dos pólipos e do câncer colorretal $^{(3)}$.

O estudo em questão tem o objetivo de analisar a indicação do exame, a localização e os resultados anatomopatológico de 100 polipectomias colonoscópicas realizadas no Setor de Endoscopia da Faculdade de Medicina de Botucatu, durante os anos de 2002 e 2003.

\section{MATERIAL E MÉTODOS}

Estudamos retrospectivamente a indicação do exame, o resultado anatomopatológico e a localização dos pólipos retirados por polipectomias realizadas em nosso serviço (independentemente da técnica utiliza- da) no período que compreendeu os anos de 2002 e 2003. Para isso, selecionamos os prontuários de 75 pacientes submetidos ao procedimento no referido período.

Foram analisados os dados relativos ao paciente e ao exame: idade, sexo, procedência, história de sangramento prévio, indicação do procedimento (diagnóstica, terapêutica ou controle), presença de doença inflamatória intestinal associada, antecedentes pessoais de adenocarcinoma no cólon. Adicionalmente, caracterizamos aspectos pertinentes ao(s) pólipo(s): resultado anatomopatológico (incluindo grau de atipia), número de pólipos retirados, localização e se os mesmos representavam ou não recidivas. Embasados nestas informações, foi preenchido o protocolo sendo que ao final da coleta dos dados, procedeu-se à análise estatística dos resultados.

\section{RESULTADOS}

Dentre os 75 pacientes cujos prontuários foram analisados, 18 indivíduos foram submetidos à colonoscopia 2 vezes durante o referido período de estudo (2002-2003), 2 deles o foram 3 vezes e 4 o foram 4 vezes, totalizando 100 colonoscopias. As idades dos pacientes variaram entre 6 e 92 anos, sendo que a média e desvio padrão foram, respectivamente, 56,3 e $(+/-) 16,7$ anos.

Observou-se que a prevalência de pólipos colônicos cresce com o aumento da idade (Tabela 1), havendo uma distribuição equilibrada entre os sexos feminino $(50,6 \%)$ e masculino $(49,4 \%)$.

Verificamos que em 46 pacientes $(61,4 \%)$ havia história previa de sangramento, e que 21 pacientes (28\%) apresentavam antecedente pessoal de pólipo ou câncer (Tabela 2).

Na Tabela 3 é possível verificar que na maior parte dos indivíduos havia um único pólipo, e que somente em 9 dos 100 procedimentos (9\%) havia mais do que 5 pólipos.

A distribuição dos pólipos está demonstrada no Gráfico 1, onde é possível constatar que a localização preferencial é nos segmentos distais.

Tabela 1 - Distribuição dos pólipos segundo o número de pacientes. Freqüência absoluta e relativa.

\begin{tabular}{lcccc}
\hline & $=\mathbf{4 0}$ anos $(\%)$ & $\mathbf{4 0 - 6 0}$ anos $(\%)$ & $=60$ anos $(\%)$ & Total $(\%)$ \\
\cline { 2 - 5 } Número de pessoas $(\%)$ & $14(18,6)$ & $28(37,3)$ & $32(44,1)$ & $75(100)$ \\
\hline
\end{tabular}


Tabela 2 - Correlação entre antecedente pessoal (AP) de adenocarcinoma em cólon elou reto e número de pacientes. Freqüência absoluta e relativa.

\begin{tabular}{lccc}
\hline & Sim $(\%)$ & Não (\%) & Total (\%) \\
\cline { 2 - 4 } AP adenocarcinoma & $21(28)$ & $54(72)$ & $75(100)$ \\
\hline
\end{tabular}

A análise das características histológicas permite constatar que, em sua maioria, os pólipos encontrados foram os adenomatosos (54\%), seguidos dos hiperplásicos $(17,8 \%)$, inflamatórios $(10 \%)$ e juvenis (3\%) (Gráfico 2).

A tabela 4 e o Gráfico 3 sumarizam as características dos pólipos adenomatosos encontrados no que tange, respectivamente, à gravidade das atipias (leve, moderada ou grave) e tipo histológico (tubular, tubuloviloso e viloso).

\section{DISCUSSÃO}

Relatos da literatura demonstram que os pólipos são encontrados com maior frequiência em homens, sendo que a relação entre o sexo masculino e feminino é aproximadamente 1.4:1 $1^{(8)}$ fato este não observado em nosso estudo, onde constatamos que esta proporção é de 1:1.

Pólipos adenomatosos e hiperplásicos responderam, respectivamente, por $54 \%$ e $17,8 \%$, totalizando $71,8 \%$. Tais resultados são concordantes com a literatura, onde se evidencia o predomínio de leões adenomatosas sobre as hiperplásicas ${ }^{(7,9,10)}$. Quanto a estas últimas, apesar de tradicionalmente consideradas não neoplásicas, foi observado que algumas mutações genéticas estão envolvidas no aparecimento deste tipo histológico.

Observamos que a distribuição dos pólipos é crescente no sentido distal, sendo alta a porcentagem de pólipos retais (51\%). Este resultado é consistente com muitos outros estudos ${ }^{(11,12)}$. IKEDA e $\operatorname{cols}^{(6)}$ demonstraram ainda que a incidência de câncer é significativamente maior em sítios mais distais do cólon e do reto quando comparada à distribuição dos pólipos adenomatosos, sugerindo que estes teriam maior potencial de malignidade quando localizados nestes segmentos mais distais.

No que diz respeito aos antecedentes de sangramento gastrintestinal baixo, sabe-se que esta condição tem inúmeras causas possíveis, sendo as mais comuns as afecções anorretais, como as

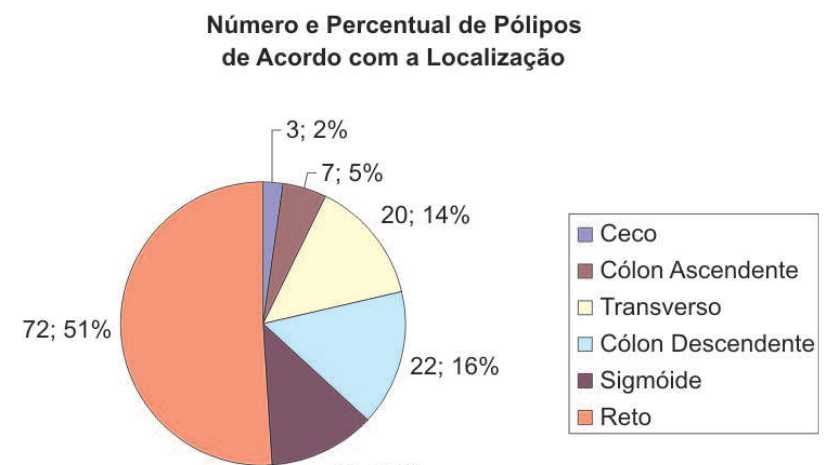

Gráfico 1 - Distribuição dos pólipos de acordo com a localização. Freqüiência relativa (\%).

Número e Percentual de Pólipos de Acordo com o Tipo Histológico

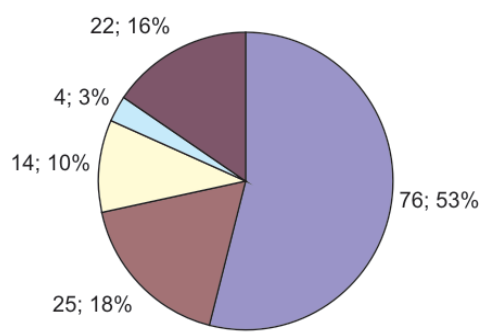

$\square$ Adenomatoso

$\square$ Hiperplásico

$\square$ Inflamatório

$\square$ Juvenil

Não Informado

Gráfico 2 - Distribuição dos pólipos de acordo com o tipo histológico. Freqüência relativa (\%).

Percentual de Adenomas de Acordo com o Subtipo Histológico

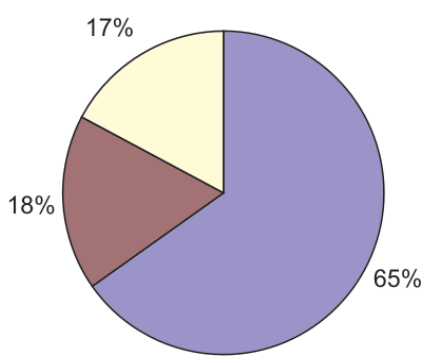

$\square$ Tubular

$\square$ Tubuloviloso

$\square$ Viloso

Gráfico 3 - Distribuição dos pólipos adenomatosos de acordo com seu subtipo histológico. Freqüência relativa (\%). 
Tabela 3 - Correlação entre número de pólipos encontrados por colonoscopia e número de procedimentos colonoscópicos realizados.

$\mathbf{N}^{0}$ de pólipos encontrados

\begin{tabular}{lcccc}
\hline & $\mathbf{1}$ & $\mathbf{2 - 5}$ & Múltiplos & Total \\
\cline { 2 - 5 } Procedimentos & $63 \%$ & $28 \%$ & $9 \%$ & $100 \%$ \\
\hline
\end{tabular}

Tabela 4 - Distribuição dos pólipos adenomatosos de acordo com gravidade das atipias.

\begin{tabular}{lcccc}
\hline & AT leves & AT moderadas & AT graves & Total \\
\cline { 2 - 5 }$\%$ adenomas & 78 & 15 & 7 & 100 \\
\hline
\end{tabular}

$A T=$ atipias

hemorróidas ${ }^{(8,13)}$. Neoplasias colônicas causam aproximadamente $20 \%$ dos episódios de sangramento do trato gastrointestinal baixo ${ }_{2}^{(10)}$ havendo ainda, grande discordância entre diferentes autores no que se refere ao exato mecanismo responsável pelo sangramento de um pólipo ${ }^{(14)}$. No presente estudo, os dados pertinentes à presença de sangramento prévio à realização da colonoscopia foram coletados a partir das queixas dos pacientes anotadas em seus prontuários; não foram pesquisadas outras possíveis causas de sangramento. Este fato limita a validade dos dados com relação à prevalência de sintomas em pacientes acometidos por pólipos no nosso estudo, mas pode ser relevante como dado da história que viabilize a indicação de uma colonoscopia.

Dentre os adenomas encontrados, o padrão tubular foi o mais freqüente (65\%), enquanto o viloso foi o menos comum (17\%). Nossos pacientes tiveram os pólipos adenomatosos classificados na maneira habitual, de modo que a análise histológica não inclui alguns padrões mais recentemente descritos (como os serrilhados e os microtubulares). Embora tenhamos observado incidências diferentes para cada tipo histológico, a literatura consultada mostrou predomínio de lesões tubulares ${ }^{(7-10)}$, concordando com os resultados obtidos.

\section{CONCLUSÃO}

Constatamos que, em nossa casuística, a maioria dos pólipos colorretais são adenomatosos e de localização distal. Dentre os adenomas, a maior proporção é representada por pólipos do tipo tubular. A colonoscopia é considerada um método ótimo para detecção de pólipos colônicos, tendo em vista a possibilidade de realizar-se polipectomias terapêuticas. Pequenos pólipos são fáceis de remover e a remoção colonoscópica raramente é seguida de alguma complicação. Adicionalmente, sabe-se que pacientes com múltiplos adenomas ou pólipos adenomatosos de grande diâmetro apresentam alto risco de desenvolver câncer colorretal. Estes fatores, somados à detecção de mutações na gênese dos pólipos hiperplásicos, tornam a colonoscopia um procedimento custo-efetivo, e um excelente método diagnóstico e terapêutico.

\footnotetext{
ABSTRACT: Objective: To analyze the retrospective results of the anatomopathological study of colonoscopic polypectomies performed at the Sector of Endoscopy of the School of Medicine of Botucatu - UNESP during the years of 2002 and 2003. Material and Methods: We studied in retrospect the characteristics of the polyps found and removed during colonoscopies performed at our service, including: size, site of appearance and distribution, as well as the reasons for removal and the anatomopathological results. Results: In 100 polypectomies performed in $\mathbf{7 5}$ patients we observed that $63 \%$ of the polyps were found in the area comprised by the sigmoid and the rectum and $15,8 \%$ were found in the left colon; the anatomopathological results showed that most of them had adenomatous features $(54 \%)$. The patients' age varied between 6 and 92 years, with no predominance of any sex; the main indication for the colonoscopies was history of bleeding. Conclusion: We verified the important role of colonoscopies in diagnosing and treating the colorectal cancer; the results found showed that most of colonic polyps are adenomatous, small and distal in their localization.
}

Key words: colonic polyps, adenomatous polyps, colonoscopy, colorectal neoplasms, histology. 


\section{REFERÊNCIAS}

1. Leslie A, Carey FA, Pratt NR, Steele RJC. The colorectal adenoma-carcinoma sequence. Br J Surg 2002; 89: 845-9.

2. Rocha BP, Ferrari AP, Forones NM. Frequiência de pólipos em doentes operados de Câncer colorretal Arq. Gastroenterol 2000; 37 (1): 31-4. disponível em http://www.sciello.br.

3. Chen Liu, James M, Crawford, The gastrointestinal tract in: Kumar V, Abas AK, Fausto N, Robbins and Cotran pathologic basis of disease, 7th ed Philadelphia: Elservia Saunders, 2005 p.857-64.

4. Church JM. Clinical significance of small colorectal polyps. Dis Colon Rectum 2004; 47(4): 481-5.

5. Jass, JR. Hyperplastic polyps of the colorrectum-innocent or guilty? Dis Colon Rectum 2001; 44:163-6.

6. Ikeda Y, Mori M, Yoshizumi T, Sugimachi K. Cancer and adenomatous polyp distribution in the colorectum. Am J Gastroenterol 1999; 94:191-3.

7. Manzione CR, Nadal SR, Nadal MA, Melo SMV. Análise morfológica e histológica de pólipos colorretais submetidos à ressecção endoscópica. Rev Bras de Coloproct 2004; 24(2):119-25.

8. Shennak MM, Tarawneh MM. Pattern of colonic disease in lower gastrointestinal bleeding in Jordanian patients: a prospective colonoscopy study. Dis Colon Rectum 1997; 40:208-14.
9. Webb WA, McDaniel L, Jones L. The anatomical distribution of colorectal polyps at colonoscopy. Arch Intern Med 2003; 163:413-20.

10. Weston AP, Campbell DR. Diminutive colonic polyps: histopathology, spatial distribution, concomitant significant lesions, and treatment complications. Am J Gastroenterol 1995; 90:24-8.

11. Burt RW, Samowitz WS. The adenomatous polyps and the hereditary polyposis syndromes. Gastroenterol Clin North Am 1988; 17:657-78.

12. Welch CE, Hedberg SE. Polypoid lesions of the gastrointestinal tract. $2^{\text {nd }}$ ed. Philadelphia: WB Saunders, 1975.

13. Vernava AM, Moore BA, Longo WE, Johnson FE. Lower gastrointestinal bleeding. Dis Colon Rectum 1997; 40:846-58.

14. Uno Y, Munakata A. Endoscopic an histologic correlates of colorectal polyp bleeding. Gastrointest Endosc 1995; 41 (5): 460-7.

\section{Endereço para Correspondência:} ROGÉRIO SAAD HOSSNE

Departamento de Cirurgia e Ortopedia

Faculdade de Medicina de Botucatu - UNESP

Distrito de Rubião Júnior $\mathrm{s} / \mathrm{n}^{\circ}$

18618-000 Botucatu - SP

e-mail: saad@fmb.unesp.br

Fax: (14) 3815-7428 